The Nepali Mathematical Sciences Report, Vol. 37, No. 1 \& 2, 2020: 86-95

DOI:: https://doi.org/10.3126/nmsr.v37i1-2.34098

\title{
A COMPARISON OF CLASSICAL HOLOMORPHIC DYNAMICS AND HOLOMORPHIC SEMIGROUP DYNAMICS-II
}

\author{
BISHNU HARI SUBEDI ${ }^{1}$ AND AJAYA SINGH ${ }^{2}$ \\ ${ }^{1,2}$ Central Department of Mathematics, Institute of Science and Technology, Tribhuvan \\ University, Kirtipur, Kathmandu, Nepal \\ Emails of Corresponding Author: subedi.abs@gmail.com, subedi_bh@cdmathtu.edu.np
}

\begin{abstract}
In this paper, we prove that the escaping set of a transcendental semigroup is S-forward invariant. We also prove that if a holomorphic semigroup is abelian, then the Fatou, Julia, and escaping sets are S-completely invariant. We also investigate certain cases and conditions that the holomorphic semigroup dynamics exhibits the similar dynamical behavior just like a classical holomorphic dynamics. Frequently, we also examine certain amount of connections and contrasts between classical holomorphic dynamics and holomorphic semigroup dynamics.
\end{abstract}

Key Words:Holomorphic semigroup, transcendental semigroup, Fatou set, Julia set, escaping set, Scompletely invariant set

AMS (MOS) [2010] Subject Classification. 37F10, 30D05

\section{INTRODUCTION}

In the paper [12], we compared some basic structure and properties of Fatou, Julia, and escaping sets in classical holomorphic dynamics and holomorphic semigroup dynamics. We found that most of the properties in classical cases were preserved and generalized in case of semigroups. However, there are few results in classical cases, which were not preserved and generalized in case of semigroups. For example, an escaping set in holomorphic semigroup dynamics may be empty but it is non-empty in classical case, and Julia set may not be the whole complex plane or the Riemann sphere when it has interior points. For an entire semigroup $S, V-f(U)$, where $V$ be a Fatou component containing $f(U)$ for some $f \in S$ may be unbounded but in classical case, $V-f(U)$ contains at most one point, and hence bounded if $f$ is an entire function. In this paper, we continue our study of comparing properties and structure of these sets in both classical and semigroup cases. Basically, we concentrate more on the invariant features of these sets.

Dynamical study of holomorphic semigroups were first studied by Hinkannen and Martin [4] in the case of rational semigroups, and by Poon [9] in the case of transcendental semigroups. We follow their concepts and definitions as usual. In this paper, we set up 
these concepts and definitions as follows. Let $A$ be a set of holomorphic functions on $\mathbb{C}$ (or on $\mathbb{C}_{\infty}$ or on certain subsets thereof). Then we can construct a semigroup $S$ consisting of all elements that can be expressed as a finite composition of the elements in $A$. We call such a semigroup $S$ is a holomorphic semigroup generated by the set $A$. For simplicity, we denote the class of all rational functions on $\mathbb{C}_{\infty}$ by $\mathscr{R}$ and the class of all entire functions (polynomials and transcendental entire) on $\mathbb{C}$ by $\mathscr{E}$. Let $\mathscr{F}=\left\{f_{\alpha}\right\}_{\alpha \in \Delta} \subseteq \mathscr{R}$ or $\mathscr{E}$. The holomorphic semigroup generated by $\mathscr{F}$ is denoted by

$$
S=\left\langle f_{\alpha}\right\rangle
$$

The index set $\Delta$ is allowed to be infinite in general unless otherwise stated. It is easy to see that $S$ is a collection of holomorphic functions, and is closed under functional compositions. That is,

$$
f=f_{\alpha_{1}} \circ f_{\alpha_{2}} \circ f_{\alpha_{3}} \circ \cdots \circ f_{\alpha_{m}}
$$

for every $f \in S$, where $\alpha_{i} \in\{\alpha: \alpha \in \Delta\} . S$ is called a rational semigroup or an entire semigroup depending on whether $\mathscr{F} \subseteq \mathscr{R}$ or $\mathscr{F} \subseteq \mathscr{E}$. In particular, if $\mathscr{F}=\left\langle f_{\alpha}\right\rangle_{\alpha \in \Delta}$ is a collection of transcendental entire functions, then $S$ is said to be a transcendental semigroup. The holomorphic semigroup $S$ is abelian if

$$
f_{\alpha} \circ f_{\beta}=f_{\beta} \circ f_{\alpha}
$$

for all $\alpha$ and $\beta$ such that $\alpha, \beta \in \Delta$. A semigroup generated by finitely many holomorphic functions $f_{i},(i=1,2, \ldots, n)$ is called finitely generated holomorphic semigroup, and we write

$$
S=\left\langle f_{1}, f_{2}, \ldots, f_{n}\right\rangle .
$$

If $S$ is generated by only one holomorphic function $f$, then $S$ is called a cyclic semigroup, and we write

$$
S=\langle f\rangle .
$$

In this case, each $g \in S$ can be written as $g=f^{n}$, where $f^{n}$ is the $n$th iterates of $f$ with itself. It is clear that $S=\langle f\rangle$ is a trivial semigroup. The following are some algebraically sound subsets of a holomorphic semigroup.

Definition 1.1 (Subsemigroup, left ideal, right ideal and ideal). A non-empty subset $T$ of a holomorphic semigroup $S$ is called a subsemigroup of $S$ if $f \circ g \in T$ for all $f, g \in T$. A non-empty subset $I$ of $S$ is called a left (or right) ideal of $S$ if $f \circ g \in I$ (or $g \circ f \in I$ ) for all $f \in S$ and $g \in I$. $I$ is called an ideal of $S$ if it is both left and right ideal of $S$.

Every (left or right) ideal is a subsemigroup but converse may not hold. The left ideal (right ideal and an ideal) of a semigroup $S$ can be constructed easily as shown in the following assertion.

Proposition 1.1. Let $S$ be a holomorphic semigroup. For any $f \in S$, the set $S \circ f=$ $\{g \circ f: g \in S\}$ is a left ideal of $S$, the set $f \circ S=\{f \circ g: g \in S\}$ is an right ideal of $S$ and the set $S \circ f \circ S=\{h \circ f \circ g: h, g \in S\}$ is an ideal of $S$. For any left ideal $S \circ f$, there is $h \in S$ such that $(g \circ f)^{n}=h \circ f$ for all $n \in \mathbb{N}$, and for an ideal $S \circ f \circ S$, there are some $p, q \in S$ such that $(h \circ f \circ g)^{n}=p \circ f \circ q$ for all $n \in \mathbb{N}$. 


\section{Fatou, Julia, And Escaping Sets}

Definitions of the Fatou, Julia, and escaping sets of classical holomorphic dynamics can be naturally extended in the settings of holomorphic semigroups. Note that a holomorphic semigroup $S$ forms a normal family in a domain $D$ if every sequence $\left(f_{\alpha}\right) \subseteq S$ has a subsequence $\left(f_{\alpha_{k}}\right)$ which is uniformly convergent or divergent on all compact subsets of $D$. If there is a neighborhood $U$ of a point $z \in \mathbb{C}$ such that $S$ is a normal family in $U$, then we say that $S$ is normal at $z$. We say that a function $f \in S$ is iteratively divergent at $z \in \mathbb{C}$ if $f^{n}(z) \rightarrow \infty$ as $n \rightarrow \infty$. A semigroup $S$ is iteratively divergent at $z$ if every $f \in S$ is iteratively divergent at $z$. A semigroup $S$ is said to be iteratively bounded at $z$ if there is an element $f \in S$ which is not iteratively divergent at $z$.

Definition 2.1 (Fatou, Julia, and escaping sets). Fatou set of the holomorphic semigroup $S$ is defined by

$$
F(S)=\{z \in \mathbb{C}: S \text { is normal at } z\}
$$

and the Julia set $J(S)$ of $S$ is the compliment of $F(S)$. The escaping set of $S$ is defined by

$$
I(S)=\{z \in \mathbb{C}: S \text { is iteratively divergent at } z\}
$$

We call each point of the set $I(S)$ by an escaping point.

If $S=\langle f\rangle$, then the Fatou, Julia, and escaping sets are respectively denoted by $F(f), J(f)$ and $I(f)$. It is obvious that $F(S)$ is the largest open subset of $\mathbb{C}\left(\right.$ or $\left.\mathbb{C}_{\infty}\right)$ on which $S$ is normal. And its complement $J(S)$ is a closed set for any holomorphic semigroup $S$. Any maximally connected subset $U$ of the Fatou set $F(S)$ is called a Fatou component.

In [12], we prove the following two assertions as Theorems 3.1, and 3.2, which are immediate from Definition 2.1.

Theorem 2.1. Let $S$ be a holomorphic semigroup, and let $z \in \mathbb{C}$ is an escaping point under $S$. Then every sequence $\left(g_{k}\right)_{k \in \mathbb{N}}$ in $S$ is iteratively divergent at $z$.

Theorem 2.2. Let $z \in \mathbb{C}$ is an escaping point of a holomorphic semigroup $S$. Then every non-convergent sequence in $S$ has a subsequence which diverges to $\infty$ at $z$.

Note that Theorem 2.2 can be taken as an alternative definition of an escaping set, and it is also appeared in [13, Lemma 3.3].

\section{Invariant features of Fatou, Julia, and escaping sets}

The fundamental contrasts between classical holomorphic dynamics and holomorphic semigroup dynamics are appeared as different algebraic structure of corresponding semigroups. In fact, non-trivial holomorphic semigroups may not be abelian. However, a trivial semigroup is cyclic, and therefore, abelian. Note that the classical holomorphic dynamics is a dynamical study of a trivial semigroups, whereas the holomorphic semigroup dynamics is a dynamical study of a non-trivial holomorphic semigroups. The main contrast between classical complex dynamics and semigroup dynamics will appear in the invariant features of 
the Fatou, Julia, and escaping sets. Note that invariant feature is considered a very basic and fundamental structure of these sets.

Definition 3.1 (Forward, backward and completely invariant set). Let $S$ be a holomorphic semigroup. A set $U \subset \mathbb{C}$ is said to be $S$-forward invariant if $f(U) \subset U$ for all $f \in S$. It is said to be $S$-backward invariant if $f^{-1}(U)=\{z \in \mathbb{C}: f(z) \in U\} \subset U$ for all $f \in S$, and is called $S$-completely invariant if it is both S-forward and S-backward invariant.

Proposition 3.1. Let $S$ be a holomorphic semigroup. Then the Fatou set $F(S)$ is $S$-forward invariant whereas the Julia set $J(S)$ is S-backward invariant.

This Proposition 3.1 was proved by Hinkkanen and Martin [4, Theorem 2.1] if $S$ is a rational semigroup, and it was proved by Poon [9, Theorem 2.1] if $S$ is a transcendental semigroup.

Kumar and Kumar [7, Theorem 4.1] proved the following result that shows escaping set $I(S)$ is also a S-forward invariant. Here, we provide another proof based on our Definition 2.1 of escaping set.

Theorem 3.1. The escaping set $I(S)$ of a transcendental semigroup $S$ is $S$-forward invariant.

Proof. Let $z \in I(S)$. Then by Definition 2.1, semigroup $S$ is iteratively divergent at $z$, and for all $g \in S$, the subsemigroup $S \circ g=\{f \circ g: f \in S\}$ is also iteratively divergent at $z$. That is, $(f \circ g)^{n}(z) \rightarrow \infty$ as $n \rightarrow \infty$ for all $f \in S$. By Proposition 1.1, for all $n \in \mathbb{N}$, we have

$$
(f \circ g)^{n}=h_{n_{i}} \circ g
$$

for some $h_{n_{i}} \in S$ where $n_{i}$ depends on $n$. Therefore, $(f \circ g)^{n}(z) \rightarrow \infty$ as $n \rightarrow \infty \Rightarrow$ $\left(h_{n_{i}} \circ g\right)(z) \rightarrow \infty$. Then by Theorem 2.2 , there exists a subsequence $\left(h_{n_{i_{j}}} \circ g\right)_{n_{i_{j}} \in \mathbb{N}}$ of the sequence $\left(\left(h_{n_{i}} \circ g\right)\right.$ such that $\left(h_{n_{i_{j}}} \circ g\right)(z)=h_{n_{i_{j}}}(g(z)) \rightarrow \infty$ as $n_{i_{j}} \rightarrow \infty$. This proves that $S$ diverges at $g(z)$, so $g(z) \in I(S)$ for all $g \in S$. Hence $I(S)$ is a S-forward invariant.

The following example of Hinkkanen and Martin [4, Example 1] shows that the Fatou set $F(S)$ need not be a S-backward invariant, and the Julia set $J(S)$ too need not be a S-forward invariant.

Example 3.1. For a rational semigroup $S=\left\langle z^{2}, z^{2} / a\right\rangle$, where $a \in \mathbb{C},|a|>1$, the Fatou set

$$
F(S)=\{z:|z|<1 \text { or }|z|>|a|\}
$$

is not a S-backward invariant and also Julia set

$$
J(S)=\{z: 1 \leq|z| \leq|a|\}
$$

too is not a S-forward invariant. 
Fatou [2] and Julia [5] independently proved that the Fatou and Julia sets of a rational function are completely invariant. Note that all the three sets $F(f), J(f)$ and $I(f)$ of a transcendental entire function are also completely invariant. This is a fundamental contrast between classical holomorphic dynamics and the holomorphic semigroup dynamics.

We prove, under certain conditions, the Fatou set of a holomorphic semigroup is Sbackward invariant, whereas the Julia set is S-forward invariant.

Theorem 3.2. Let $S$ be an abelian holomorphic semigroup. Then the Fatou set $F(S)$ is $S$-backward invariant, and the Julia set $J(S)$ is $S$-forward invariant.

Proof. We prove that if $g(z) \in F(S)$, then $z \in F(S)$ for all $g \in S$. This follows that $g^{-1}(F(S)) \subset F(S)$ for all $g \in S$. Suppose $g(z) \in F(S)$. Let $U$ be a neighborhood of $g(z)$ such that $\bar{U} \subset F(S)$. Then there is a subsequence $\left(f^{n_{j}}\right)$ such that $f^{n_{j}}(g(z)) \rightarrow f(g(z))$ uniformly on $U$, where $f$ is a holomorphic function or constant $\infty$. Since $S$ is abelian, so we have $g\left(f^{n_{j}}(z)\right) \rightarrow g(f(z))$ uniformly on $U$. This shows that $g \circ f^{n_{j}} \rightarrow g \circ f$ uniformly on $U$. This proves that $z \in F(S)$ for all $g \in S$.

From Theorems 3.1 and 3.2 , we can say that the Fatou set $F(S)$ and the Julia set $J(S)$ are S-completely invariant if $S$ is an abelian holomorphic semigroup. The following example of Hinkkanen and Martin [4, Example 2] is based on Theorem 3.2 .

Example 3.2. The semigroup $S=\left\langle T_{n}(z): n=0,1,2, \ldots\right\rangle$ generated by Tchebyshev polynomials $T_{n}(z)$ defined by $T_{0}(z)=1, T_{1}(z)=z$ and $T_{n+1}(z)=2 z T_{n}(z)-T_{n-1}(z)$ is abelian. Therefore, by Theorem 3.2 , Fatou set $F(S)$ is S-forward invariant and Julia set $J(S)$ is S-backward invariant.

Kumar and Kumar [8, Theorem 2.1] proved the following assertion for backward invariance of $I(S)$. Here, we give another proof based on Definition 2.1 of an escaping set.

Theorem 3.3. The escaping set $I(S)$ of a transcendental semigroup $S$ is $S$-backward invariant if $S$ is an abelian transcendental semigroup.

Proof. We prove that if $g(z) \in I(S)$, then $z \in I(S)$ for all $g \in S$. This follows that $g^{-1}(I(S)) \subset I(S)$ for all $g \in S$. This will be proved if we are able to prove its contrapositive statement: if $z \notin I(S)$, then $g(z) \notin I(S)$ for all $g \in S$. Let $z \notin I(S)$. Then there is some $f \in S$ which is iteratively bounded at $z$. That is, $f^{n}(z) \nrightarrow \infty$ as $n \rightarrow \infty$. In this case, there exists a sequence $\left(f_{k}\right)_{k \in \mathbb{N}}$ in $S$ containing $f$ which is iteratively bounded at $z$, and all subsequences of this sequence containing $f$ are also iteratively bounded at $z$. Now, for any $g \in S,\left(f_{k} \circ g\right)_{k \in \mathbb{N}}$ is a sequence in $S$. Since $S$ is abelian and $g$ is a transcendental entire function, so by the continuity of $g$ at $z \in \mathbb{C}$, we can write that

$$
\left(f_{k} \circ g\right)(z)=\left(g \circ f_{k}\right)(z)
$$

for all $k \in \mathbb{N}$. From which it follows that the sequence $\left(f_{k} \circ g\right)_{k \in \mathbb{N}}$ is iteratively bounded at $z$. Therefore, all subsequences of this sequences are iteratively bounded at $z$. From 3.1, we can say that all subsequences of the sequence $\left(f_{k} \circ g\right)_{k \in \mathbb{N}}$ are iteratively bounded at $g(z)$. 
That is, $g(z) \notin I(S)$ for all $g \in S$. Therefore, $g^{-1}(I(S)) \subset I(S)$ for all $g \in S$. This proves that $I(S)$ is a S-backward invariant.

From the above results (Theorems 3.1 and 3.3 , we can conclude that the sets $F(S)$, $J(S)$, and $I(S)$ are S-completely invariant if $S$ is an abelian transcendental semigroup. For example, the following semigroups

(1) $\langle z+\gamma \sin z, z+\gamma \sin z+2 k \pi\rangle$,

(2) $\langle z+\gamma \sin z,-z-\gamma \sin z+2 k \pi\rangle$,

(3) $\left\langle z+\gamma e^{z}, z+\gamma e^{z}+2 k \pi i\right\rangle$,

(4) $\langle z-\sin z, z-\sin z+2 \pi\rangle$,

(5) $\left\langle e^{\gamma z}, e^{\gamma z+\frac{2 \pi i}{\gamma}}\right\rangle$, where $0<\gamma<e^{-1}$,

are all abelian transcendental semigroups, so their Fatou, Julia, and escaping sets are all S-completely invariant.

Theorems 3.2 and 3.3 give a kind of connection between classical holomorphic dynamics and holomorphic semigroup dynamics. We got a completely invariant structure of the Fatou, Julia, and escaping sets in both classical holomorphic dynamics and semigroup dynamics because of their associated abelian semigroups. This was our expectation that an abelian semigroup (rational and transcendental) must exhibit similar dynamical features just like the classical holomorphic dynamics. Note that it can not be concluded that the Fatou, Julia, and escaping sets can not be completely invariant unless the semigroup is abelian.

If $S$ is a finitely generated rational semigroup, then Sumi [14, Lemma 1.1.4 (2)] proved the following result.

Proposition 3.2. If $S=\left\langle f_{1}, f_{2}, \ldots, f_{n}\right\rangle$ is a finitely generated rational semigroup, then $F(S)=\bigcap_{i=1}^{n} f_{i}^{-1}(F(S))$, and $J(S)=\bigcup_{i=1}^{n} f_{i}^{-1}(J(S))$.

In the case of finitely generated transcendental semigroup, we prove following result which is an analogous to Proposition 3.2 .

Theorem 3.4. If $S=\left\langle f_{1}, f_{2}, \ldots, f_{n}\right\rangle$ is a finitely generated transcendental semigroup, then $F(S)=\bigcap_{i=1}^{n} f_{i}^{-1}(F(S))$ and $J(S)=\bigcup_{i=1}^{n} f_{i}^{-1}(J(S))$.

Proof. The Fatou set $F(S)$ is S-forward invariant in general (Theorem 3.2 ). Hence, $f_{i}(F(S)) \subset$ $F(S)$, and it follows that $F(S) \subset \bigcap_{i=1}^{n} f_{i}^{-1}(F(S))$ for all $i$.

Next, let $z_{0} \in \bigcap_{i=1}^{n} f_{i}^{-1}(F(S))$. Then (say) $w_{i}=f_{i}\left(z_{0}\right) \in F(S)$ for all $i$. The semigroup $S$ is normal at $w_{i}$ for all $i$. In other words, every $g \in S$ is equicontinuous at $w_{i}$ for all $i$. That is, for any $\epsilon>0$, there is $\delta>0$ such that $d\left(g(w), g\left(w_{i}\right)\right)<\epsilon$, whenever $d\left(w, w_{i}\right)<$ $\delta$ for all $w \in F(S)$ and $i=1,2,3, \ldots, n$, where $d$ represents the Euclidean metric on $\mathbb{C}$. For such $\delta$, there is $\eta>0$ such that $d\left(f_{i}(z), f_{i}\left(z_{0}\right)\right)<\delta$, whenever $d\left(z, z_{0}\right)<\eta$ for all $z \in \bigcap_{i=1}^{n} f_{i}^{-1}(F(S))$ and $i=1,2,3, \ldots, n$. Thus, ultimately, we conclude that $d\left(g\left(f_{i}(z)\right), g\left(f_{i}\left(z_{0}\right)\right)\right)<\epsilon$ whenever $d\left(z, z_{0}\right)<\eta$ for all $z \in \bigcap_{i=1}^{n} f_{i}^{-1}(F(S))$ and $i=$ $1,2,3, \ldots, n$. We can also write $S=\bigcup_{i=1}^{n}\left(S \circ f_{i}\right)$. So, $S$ is equicontinuous at $z_{0}$. That is, 
$z_{0} \in F(S)$. Hence, $F(S)=\bigcap_{i=1}^{n} f_{i}^{-1}(F(S))$. The second part of this theorem easily follows easily since

$$
J(S)=\mathbb{C}-F(S)=\mathbb{C}-\bigcap_{i=1}^{n} f_{i}^{-1}(F(S))=\bigcup_{i=1}^{n}\left(\mathbb{C}-f_{i}^{-1}(F(S))=\bigcup_{i=1}^{n} f_{i}^{-1}(J(S)) .\right.
$$

The relation $J(S)=\bigcup_{i=1}^{n} f_{i}^{-1}(J(S))$ for the Julia set is called a backward self similarity. With this property, dynamics of a holomorphic semigroup can be regarded as backward iterated function system-BIFS associated to the set $\left\{f_{1}, f_{2}, \ldots, f_{n}\right\}$ of generators of semigroup $S$. The Julia set $J(S)$ is considered as an attractor or invariant for BIFS. Indeed, $J(S)$ is a unique attractor, which is usually a fractal.

Theorems 3.1 and 3.3 can be used directly to express the escaping set $I(S)$ as a finite intersection of all pre-images of itself under the generators of the semigroup $S$ if it is finitely generated. The following result is due to Kumar and Kumar [8, Theorem 2.6]. Here, we give an alternative proof.

Theorem 3.5. If $S=\left\langle f_{1}, f_{2}, \ldots, f_{n}\right\rangle$ is a finitely generated abelian transcendental semigroup, then $I(S)=\bigcap_{i=1}^{n} f_{i}^{-1}(I(S))$ if $S$ is an abelian semigroup.

Proof. $I(S)$ is completely invariant under the assumption of the theorem (see for instance Theorems 3.1, 3.3). So, we have $f_{i}(I(S)) \subset I(S)$ and $f_{i}^{-1}(I(S)) \subset I(S)$ for all $1 \leq i \leq n$. From which we get respectively

$$
I(S) \subset \bigcap_{i=1}^{n} f_{i}^{-1}(I(S)) \text { and } I(S) \supset \bigcap_{i=1}^{n} f_{i}^{-1}(I(S))
$$

for all $1 \leq i \leq n$. Thus, we get $I(S)=\bigcap_{i=1}^{n} f_{i}^{-1}(I(S))$.

\section{FurThER COMPARISON OF CLASSICAL DYNAMICS AND SEMIGROUP DYNAMICS}

There are certain holomorphic semigroups where semigroup dynamics behave in the same way as in classical holomorphic dynamics. That is, there are certain holomorphic semigroups whose Fatou, Julia, and escaping sets are same as Fatou, Julia, and escaping sets of each of its generators. From the following result of Kumar and Kumar [7, Theorem 3.4], we get such a conclusion.

Proposition 4.1. Let $S=\langle f, g\rangle$ be a transcendental semigroup generated by periodic function $f$ with period $p$ and another function $g$ defined by $g=f^{n}+p, n \in \mathbb{N}$. Then $F(S)=F(f), J(S)=J(f)$ and $I(S)=I(f)$.

Note that in case of a semigroup stated in Proposition 4.1, each of the Fatou, Julia, and escaping set is a S-completely invariant. We prove the following Theorem which gives similar result in case of rational semigroup.

Theorem 4.1. Let $S$ be an abelian rational semigroup. Then $J(S)=J(f)$ and $F(S)=$ $F(f)$ for all $f \in S$ of degree at least two. 
We recall the following results of Fatou [3] and Julia [6] concerning commuting rational functions, and Hinkkanen and Martin ([4, Lemma 3.1 and Corollary 3.1]) concerning the rational semigroups.

Proposition 4.2. Let $f$ and $g$ be two rational functions of degree at least two such that $f \circ g=g \circ f$. Then $J(f)=J(g)$.

Proposition 4.3. Let $S$ be a rational semigroup. Then Julia set $J(S)$ is perfect and $J(S)=$ $\overline{\bigcup_{f \in S} J(f)}$.

These results will be used in the proof of Theorem 4.1.

Proof of Theorem 4.1. Since semigroup $S$ is abelian, so we have $f_{i} \circ f_{j}=f_{j} \circ f_{i}$ for all generators $f_{i}$ and $f_{j}$ with $i \neq j$. Then by Proposition 4.2, $J\left(f_{i}\right)=J\left(f_{j}\right)$ for all $i$ and $j$ with $i \neq j$. Also, every $f \in S$ permutes with each generator $f_{i}$ for all $i$, so again by the same Proposition 4.2, $J(f)=J\left(f_{i}\right)$ for all $i$. This fact, together with Proposition 4.3, we can conclude that $J(S)=J(f)$ for all $f \in S$.

The analogous result in a transcendental semigroup may not hold, in general, because Proposition 4.2 is still unanswered in case of permutable transcendental entire functions. Julia sets for two permutable entire functions were studied in [10, 11, 15], where under certain conditions we can get results similar to Proposition 4.2 . If we impose extra conditions in Theorem 4.1, then the result analogous to the Theorem 4.1 holds in case of transcendental semigroups. One of the analogous result of Theorem 4.1 was proved by Poon [9, Theorem 5.1]. Here, we only give sketch of the proof similar to the proof of Theorem 4.1 .

Recall that

$$
C V(f)=\left\{w \in \mathbb{C}: w=f(z) \text { for some } z \text { such that } f^{\prime}(z)=0\right\}
$$

is the set of critical values. The set $A V(f)$ consisting of all $w \in \mathbb{C}$ such that there exists a curve $\Gamma:[0, \infty) \rightarrow \mathbb{C}$ so that $\Gamma(t) \rightarrow \infty$ and $f(\Gamma(t)) \rightarrow w$ as $t \rightarrow \infty$ is the set of asymptotic values of $f$ and

$$
S V(f)=\overline{(C V(f) \cup A V(f))}
$$

is the set of singular values of $f$. If $S V(f)$ finite, then $f$ is said to be of finite type. If $S V(f)$ bounded, then $f$ is said to be of bounded type. The sets

$$
\mathscr{S}=\{f: f \text { is of finite type }\} \text { and } \mathscr{B}=\{f: f \text { is of bounded type }\}
$$

are respectively called Speiser class and Eremenko-Lyubich class.

We recall the following three results of Poon [9, Theorems 4.1, 4.2 and Lemmas 5.1, 5.2 ] for our reference.

Proposition 4.4. Let $S$ be a transcendental semigroup. Then Julia set $J(S)$ is perfect and $J(S)=\overline{\bigcup_{f \in S} J(f)}$.

Proposition 4.5. Let $f$ and $g$ be two transcendental entire functions of finite type. Then $f \circ g$ is of finite type. Moreover, if $f$ and $g$ are permutable, then $J(f)=J(g)$. 
Proposition 4.6. Let $S$ be an abelian transcendental semigroup in which each generator is of finite type. Then $J(S)=J(f)$ for all $f \in S$.

Proof of Proposition 4.6. We have given that semigroup $S$ is abelian. Then by Proposition 4.5. $J\left(f_{i}\right)=J\left(f_{j}\right)$ for all generators $f_{i}$ and $f_{j}$ with $i \neq j$. Each

$$
f=f_{i_{1}} \circ f_{i_{2}} \circ \ldots f_{i_{m}}
$$

is of finite type and $J(f)=J\left(f_{i}\right)$ for all $i$. This fact together with Proposition 4.4, we can conclude that $J(S)=J(f)$ for all $f \in S$.

We prove the following assertion that the condition mentioned in the Proposition 4.6 is sufficient to show $I(S)=I(f)$ for all $f \in S$.

Theorem 4.2. Let $S$ is an abelian transcendental semigroup in which each generator is of finite type. Then $I(S)=I(f)$ for all $f \in S$.

Lemma 4.1. If $f$ and $g$ are permutable transcendental entire functions of finite type, then $I(f)=I(g)$.

Proof. As given in the statement, Poon [9, Lemma 5.2] showed that $F(f)=F(g)$ (Proposition 4.6). Eremenko and Lyubich [1 proved that if transcendental function $f \in \mathscr{S}$, then $I(f) \subset J(f)$, and $J(f)=\overline{I(f)}$. For any function of finite type, we must have $\overline{I(f)}=\overline{I(g)}$. This Lemma will be proved if we show $J(f)-I(f)=J(g)-I(g)$. Let $z \in J(f)-I(f)$. Then $z$ is a non-escaping point of $J(f)$ and so the sequence $\left(f^{n}\right)$ has a bounded subsequence at $z . J(f)=J(g)$ implies that the sequence $\left(g^{n}\right)$ has also a bounded subsequence at $z$. So $z \in J(g)-I(g)$. Therefore, $J(f)-I(f) \subset J(g)-I(g)$. By similar argument, we can show that $J(g)-I(g) \subset J(f)-I(g)$. Hence, our claim is established.

Proof of Theorem 4.2. The semigroup $S$ is abelian, so we have $f_{i} \circ f_{j}=f_{j} \circ f_{i}$ for all generator $f_{i}$ and $f_{j}$ with $i \neq j$. By Lemma 4.1, we have $I\left(f_{i}\right)=I\left(f_{j}\right)$. Any $f \in S$ can be written as

$$
f=f_{i_{1}} \circ f_{i_{2}} \circ f_{i_{3}} \circ \cdots \circ f_{i_{m}}
$$

By permutability of each $f_{i}$, we can rearrange $f_{i_{j}}$ and ultimately represented by

$$
f=f_{1}^{t_{1}} \circ f_{2}^{t_{2}} \circ \ldots \circ f_{n}^{t_{n}}
$$

where each $t_{k} \geq 0$ is an integer for $k=1,2, \ldots, n$. Lemma 4.5 can be applied repeatably to show that each of $f_{1}^{t_{1}}, f_{2}^{t_{2}}, \ldots, f_{n}^{t_{n}}$ is of finite type, and so $f$ is itself finite type. Since each $f_{i}$ permutes with $f$, and hence by Lemma 4.1. $I\left(f_{i}\right)=I(f)$ for all $f \in S$. Therefore, $I(S)=I(f)$ for each $f \in S$.

Acknowledgements: We express our heartfelt thanks to anonymous referees for their valuable suggestions and comments over the draft of this manuscript.

This research work of the first author is supported by a PhD faculty fellowship-2070 of the University Grants Commission, Nepal and a NMS Nick-Simon Fellowship- 2018 of Nepal Mathematical Society. The first author also like to thank CIMPA (France) and 
CRM (Spain) for CIMPA-CARMIN fellowship (round trip plane fare, accommodations with monthly stipends) to take part IRP Low Dimensional Dynamical Systems and Application at Centre de Recerca Matematica (CRM), Barcelona, Spain.

\section{REFERENCES}

[1] A. Eremenko and M. Y. Lyubich, Dynamical properties of some classes of entire functions. Ann. Inst. Fourier, Grenoble, 42 (1992): 989-1020.

[2] P. Fatou, Sur les equations fonctionelles. Bull. Soc. Math. France, 47 (1919), 161-271.

[3] P. Fatou, Sur les iterations analytique et les substitutions permutables. J. Math. 2 (1923): 343-384.

[4] A. Hinkkanen and G. J. Martin, The dynamics of semigroups of rational functions- I. Proc. London Math. Soc. (3) 73 (1996): 358-384.

[5] G. Julia, Memoire sur l'iteration des fractions rationelles. J. Math. Pures Appl. 1 (8) (1918): 47-245.

[6] G. Julia, Memoire sur la permutabilite des fractions rationalles. Ann. Sci. Ecole Norm. Sup. 39 (3) (1922): 131-215.

[7] D. Kumar and S. Kumar, The dynamics of semigroups of transcendental entire functions-II. Indian J. Pure Appl. Math. 47 (3) (2016): 409-423. arXiv: 1401.0425 v3.

[8] D. Kumar and S. Kumar, Escaping set and Julia set of transcendental semigroups. Advances in Theo. and Appl. Math. 11 (4): 423-436. arXiv:141.2747 v3.

[9] K.K. Poon, Fatou-Julia theory on transcendental semigroups. Bull. Austral. Math. Soc., 58 (1998): 403-410.

[10] K.K. Poon and C.C. Yang, Dynamical behavior of two permutable entire functions. Annales Polonici Mathematici, LXVIII, 2 (1998): 159-163.

[11] A.P. Singh and Y. Wang, Julia sets of permutable holomorphic maps. Sciences in China, Series A: Mathematics, 49 (11) (2006): 1715-1721.

[12] B.H. Subedi and A. Singh, A short comparison of classical complex dynamics and holomorphic semigroup dynamics. arXiv:1803.08368v1 [math. DS], 2018.

[13] B. H. Subedi and A. Singh, Fatou, Julia, and escaping sets in holomorphic (sub)semigroup dynamics. Turk. J. Math., 43 (2)(2019): 930-940. DOI: 10. 3906/mat-1810-133. arXiv: 1807.04499v1 [math. DS].

[14] H. Sumi, On dynamics of hyperbolic rational semigroup. J. Math. Kyoto Univ. (JMKYAZ), 374 (1998): 717-733.

[15] W. Ng Tuen, Permutable entire functions and their Julia sets. Math. Proc. Camb. Phil. Soc., 131 (2001): 129-138. 